\title{
Modificação da escala de classificação por postos utilizada em Análise Conjunta para aprimorar o modelo obtido por regressão com variáveis dummy.
}

\author{
Anderson Laécio Galindo Trindade (USP) anderson.trindade@poli.usp.br \\ Roberto Gilioli Rotondaro (USP)
}

\begin{abstract}
Resumo
A Análise Conjunta é uma técnica para a medida de preferências que considera que o processo de escolha é determinado por utilidades associadas a níveis de um conjunto de atributos. Para a estimativa das utilidades parciais e totais, a regressão vem sendo amplamente utilizada e o seu uso direto com o ordenamento dos estímulos em postos também tem sido empregado. Este trabalho propõe a modificação da escala por postos fornecida pelo entrevistado em uma nova escala, ainda discreta, que mantenha a posição relativa entre os produtos originalmente ordenados. O objetivo desta mudança é estimar um modelo em que a reconstrução da ordem original seja mais eficiente. Os resultados mostram que é possível obter uma maior concordância com o ordenamento original usando o método aqui proposto, o que pode credenciar seu uso para o tratamento posterior dos dados coletados.

Palavras chave: Análise Conjunta, Classificação por Postos, Regressão.
\end{abstract}

\section{Introdução}

O comportamento de compra do consumidor inclui um conjunto de atividades que precedem, acompanham e seguem as decisões da aquisição, e ao longo das quais o indivíduo ou a organização intervém ativamente com o objetivo de efetuar escolhas com conhecimento de causa, e não de forma aleatória. O comprador é continuamente confrontado com múltiplas decisões a tomar, cuja complexidade varia segundo o tipo de produto e situação de aquisição (LAMBIN, 2000).

A Análise Conjunta é um método de análise da estrutura das preferências, que considera um produto como um conjunto de atributos. Atributos típicos são marca, cor, tamanho e preço. A variação ou os valores que cada atributo pode assumir são denominados níveis. No caso do atributo cor, azul, verde e vermelho são três níveis possíveis. Os atributos incluídos no estudo devem ser aqueles que realmente são considerados pelo comprador em sua tomada de decisão, e os níveis devem refletir os patamares existentes no mercado (AUTY, 1995).

Surgida na década de 1970, a Análise Conjunta tem se tornado uma técnica popular para a medida da preferência dos consumidores (Park, 2003), porém o seu uso tem sido diversificado. O desenvolvimento de plataformas de produtos (Moore et al., 1999), o desenvolvimento de cadeias de suprimentos (Reutterer e Kotzab, 2000), sua aplicação em marketing industrial (Auty, 1995), a identificação e interpretação de segmentos de mercado (Næs et al., 2001) são exemplos da diversidade de aplicações desta técnica.

Segundo Labin (2000), a Análise Conjunta se apoia em um conjunto de hipóteses que são resumidas a seguir:

- O entrevistado avalia o produto ou serviço como um conjunto de atributos ou características;

- quando o entrevistado avalia o produto ou serviço, associa mentalmente valores 
subjetivos a cada um dos níveis dos atributos, valores estes chamados utilidades;

- para determinar o valor da utilidade total, o entrevistado soma mentalmente os valores das utilidades parciais associadas implicitamente a cada atributo, e

- o modelo é um modelo compensatório, ou seja, o entrevistado "compensa" fracos níveis relativos a um determinado atributo por bons níveis referentes a outros.

Existem diferentes instrumentos que podem ser utilizados para medir a preferência do entrevistado, uma vez estabelecidos os atributos e níveis relevantes no estudo. A comparação emparelhada, a matriz trade-off e o perfil pleno são exemplos destes instrumentos. Os dois primeiros não refletem o processo real de decisão, pois, no mundo real, não se avaliam apenas dois produtos por vez ou apenas dois atributos isoladamente.

A escolha do perfil pleno, por sua vez, pode gerar um número muito alto de combinações (ou estímulos). Considerando um estudo com sete atributos e três níveis, existem 2187 combinações possíveis. Utilizar apenas uma fração deste número é uma consideração importante no estudo, sendo fundamental o compromisso entre um número tolerável para o entrevistado, um número adequado para o processo de estimação e o custo do estudo. A suposição de um modelo de efeitos principais, sem interações entre os atributos, permite o uso da técnica de planejamento ortogonal, o que reduz substancialmente o número de estímulos necessários.

Green e Srinivasan (1990) sugerem que o perfil pleno seja utilizado em estudos com até seis atributos. Por questões matemáticas, é desejável que o número de estímulos seja pelo menos $50 \%$ maior que o número de parâmetros estimados (Auty, 1995). Wittink et al. (1982) verificaram que atributos com maior número de níveis tem proporcionalmente mais importância que aqueles com poucos níveis, o que sugere que um número uniforme de níveis entre os diferentes atributos é desejável.

Uma vez definidos os estímulos, os entrevistados são solicitados a classificar os produtos segundo a sua preferência. Pode-se utilizar uma classificação métrica ou uma classificação não-métrica (preferencial ou por postos). Na primeira, cada produto deve ser posicionado em uma escala previamente informada ao entrevistado, que pode variar de 1 a 10 , por exemplo. $\mathrm{O}$ principal problema associado a esta classificação é a inconsistência ao logo da classificação dos estímulos, uma vez que o respondente não considera como ele classificou anteriormente um estímulo semelhante. A classificação por postos, por sua vez, também pode apresentar inconsistências caso um número elevado de estímulos seja apresentado. Uma forma de contornar este problema é solicitar ao entrevistado que classifique os estímulos em três grandes grupos de tamanho aproximado: os mais preferidos, os menos preferidos e aqueles dispostos em uma posição intermediária. Em uma segunda etapa, o entrevistado classifica os estímulos em cada um dos três grupos criados. Auty (1995) reforça que há problemas nos dois métodos e argumenta que um dos critérios para a escolha entre uma ou outra forma de classificação deve ser a receptividade dos entrevistados.

Segundo Wittink et al. (1994), em um estudo comparativo das aplicações da Análise Conjunta na Europa e nos Estados Unidos, o uso da regressão por mínimos quadrados é a técnica mais comum de estimação das utilidades, representando $59 \%$ do universo por eles pesquisado. Isso deve ocorrer devido à simplicidade de aplicação e à disponibilidade da regressão em softwares não especialistas. Apesar de ser uma técnica indicada para dados métricos, Wittink et al. citam estudos empíricos e por simulação em que a regressão é aplicada diretamente ao ordenamento por postos e fornecem resultados comparáveis aos obtidos no uso da escala métrica. Darmon e Rouziès (1999) afirmam que apesar do maior uso da escala métrica, o uso de uma ou outra classificação tem se apoiado em um conjunto restrito de evidências 
empíricas. Estes autores mostram em seu estudo que a classificação por postos é mais adequada quando se deseja o conhecimento global da função utilidade e quando é necessário interpolar valores entre níveis utilizados no estudo. Reutterer e Kotzab (2000) também aplicam a regressão à classificação por postos, justificando o uso da regressão por sua simplicidade e verificando e validando o modelo obtido por meio de estímulos adicionais, que não foram incluídos no processo de estimação.

Apresentado o contexto geral da análise conjunta, este trabalho tem por objetivo analisar um dos problemas decorrentes do uso da classificação por postos associadas à técnica de regressão, bem como propor uma modificação na escala de classificação com o objetivo de aumentar a coerência entre o ordenamento obtido através do modelo e o ordenamento original proposto pelo entrevistado. O Item seguinte apresenta o problema proposto.

\section{Um problema na classificação por postos}

Considere que a um entrevistado foi solicitado o ordenamento dos produtos $\mathrm{A}, \mathrm{B}, \mathrm{C}$ e $\mathrm{D}$, com relação a sua utilidade, conforme mostrado na Figura 1.

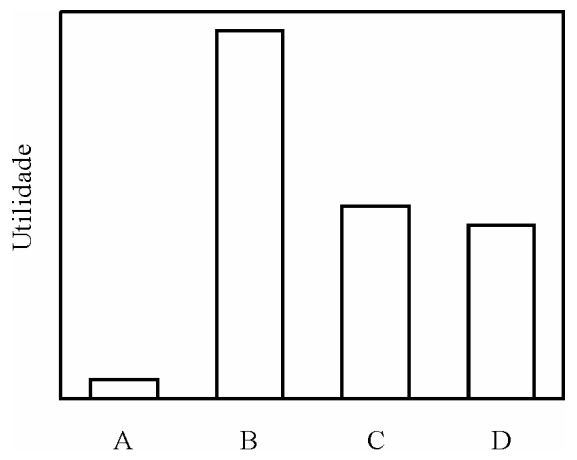

Figura 1 - Utilidade para diferentes produtos.

Caso a utilidade do entrevistado seja proporcional à altura das barras na Figura 1, a ordenação por postos, do menor para o maior, será $\{A, D, C, B\}$. Portanto, para a realização da regressão, os produtos (expressos na forma de variáveis dummy em termos nos níveis de cada atributo) serão as variáveis regressoras, enquanto o posicionamento em postos será a variável dependente, ou seja, $\{1,4,3,2\}$ (ver Malhotra, 2001). Apesar de existir uma diferença significativa na utilidade do produto $\mathrm{B}$ em relação ao produto $\mathrm{C}$, do produto $\mathrm{D}$ em relação ao produto $\mathrm{A}$ e de esta diferença ser mínima entre os produtos $\mathrm{C}$ e $\mathrm{D}$, o uso dos postos diz que a diferença percebida entre os quatro produtos é a mesma. Isso trará conseqüências nos valores das utilidades estimados, bem como na importância relativa de cada um dos fatores considerados.

Para ilustrar uma possível conseqüência, considere um estudo com 4 fatores, três níveis cada, em que os 15 estímulos mostrados na Tabela 1 são apresentados ao entrevistado e a ordenação proposta por ele também é registrada. Aplicando a regressão com variáveis dummy, calculando as utilidades de cada atributo e a utilidade global do produto obtém-se a ordem predita, também apresentada na Tabela 1 . Como pode-se notar, 9 dos 16 produtos tem uma divergência de até 2 postos, enquanto os outros 6 apresentam divergência em até 10 posições na reconstrução da ordem utilizando o modelo obtido. Esta grande divergência pode estar associada ao fato do entrevistado não ter expressado a magnitude de suas preferências, ou em outras palavras, a diferença unitária entre os postos não reflete a extensão de sua preferência.

O processo de estimação das utilidades pode ser aplicado individualmente a cada entrevistado. Um dos critérios utilizado para considerar ou não um entrevistado no estudo é o coeficiente de 
determinação do modelo obtido. Para este caso, $\mathrm{R}^{2}=0,7374$, o que para estudos deste tipo é considerado aceitável. O critério do coeficiente de determinação pode ser utilizado isoladamente, ou associado a alguma medida da divergência entre a ordem fornecida pelo entrevistado e a ordem predita pelo modelo. Uma das medidas utilizadas é o coeficiente de Kendall (Reutterer e Kotzab, 2000), que é um cálculo que mede o número de concordâncias e discordâncias entre pares de produtos, para os dois ordenamentos considerados. Há uma concordância quando, comparando-se os postos de dois produtos no ordenamento 1, verificase a mesma ordem relativa dos dois produtos no ordenamento 2 (por exemplo, Posto $_{\mathrm{A}}>$ Posto $_{\mathrm{B}}$ no ordenamento 1 e Posto $_{\mathrm{A}}>$ Posto $_{\mathrm{B}}$ no ordenamento 2). Porém, o uso de uma medida da qualidade do ordenamento, associada ao coeficiente de determinação, pode ser muito restritivo considerando situações práticas, em que o número de entrevistados é limitado por questões econômicas.

\begin{tabular}{ccccccc}
\hline Produto & Fator 1 & Fator 2 & Fator 3 & Fator 4 & $\begin{array}{c}\text { Ordenamento } \\
\text { Entrevistado }\end{array}$ & $\begin{array}{c}\text { Ordenamento } \\
\text { Previsto }\end{array}$ \\
\hline P01 & 2 & 1 & 3 & 1 & 3 & 2 \\
P02 & 1 & 2 & 3 & 1 & 10 & $5^{*}$ \\
P03 & 3 & 1 & 2 & 1 & 12 & 10 \\
P04 & 3 & 3 & 3 & 3 & 1 & 1 \\
P05 & 1 & 3 & 2 & 1 & 14 & 14 \\
P06 & 3 & 1 & 1 & 2 & 6 & 7 \\
P07 & 2 & 1 & 1 & 3 & 5 & 3 \\
P08 & 2 & 3 & 1 & 1 & 4 & $11^{*}$ \\
P09 & 2 & 2 & 2 & 2 & 13 & 15 \\
P10 & 1 & 3 & 1 & 2 & 15 & 8 \\
P11 & 1 & 1 & 2 & 3 & 8 & $6^{*}$ \\
P12 & 1 & 2 & 1 & 3 & 11 & $4 *$ \\
P13 & 1 & 1 & 3 & 2 & 9 & 9 \\
P14 & 3 & 2 & 1 & 1 & 7 & $12^{*}$ \\
P15 & 1 & 1 & 1 & 1 & 2 & 8 \\
\hline
\end{tabular}

Tabela 1 - Resposta hipotética de um entrevistado para o estímulo ortogonal de 4 fatores e 3 níveis e o ordenamento previsto pelo modelo de regressão. $\left(^{*}\right)$ indica grau de discordância superior a 3 postos.

Para aumentar o grau de concordância entre os ordenamentos, propõe-se uma modificação na escala utilizada, sem modificar a ordem relativa entre os produtos. A Figura 2 apresenta a modificação proposta para um cenário com 8 produtos. Propõe-se a modificação da escala com $n$ postos para uma escala com $2 n-1$ postos, onde $n$ é o número de produtos. Os produtos posicionados no primeiro e último postos são mantidos fixos, devendo-se determinar qual a posição dos $n$-2 produtos restantes nos postos disponíveis, mantendo-se a ordem relativa fornecida pelo entrevistado. Considerando como exemplo o produto P02 mostrado na Figura 2 , seja qual for o novo ordenamento, ele deve estar necessariamente entre os produtos P03 e P05.

A modificação proposta pode refletir a diferença de preferência não declarada por cada entrevistado. Os novos postos ocupados pelos produtos são então utilizados na determinação das utilidades por meio da regressão com variáveis dummy. O objetivo então é verificar se existe uma nova disposição dos produtos tal que seja reduzida a divergência de postos observada utilizando-se a regressão com os postos originais e que aumente o coeficiente de 
determinação originalmente obtido.

\begin{tabular}{|c|c|c|c|c|c|c|c|c|c|c|c|c|c|c|c|c|}
\hline \multicolumn{17}{|c|}{ a) ordenamento do entrevistado } \\
\hline \multirow{2}{*}{$\begin{array}{c}\text { Menos } \\
\text { preferido }\end{array}$} & \multicolumn{2}{|c|}{$\begin{array}{c}\text { Posto } \\
01\end{array}$} & \multicolumn{2}{|c|}{$\begin{array}{c}\text { Posto } \\
02\end{array}$} & \multicolumn{2}{|c|}{$\begin{array}{c}\text { Posto } \\
03\end{array}$} & \multicolumn{2}{|c|}{$\begin{array}{c}\text { Posto } \\
04 \\
\end{array}$} & $\begin{array}{c}\text { Posto } \\
05\end{array}$ & \multicolumn{2}{|c|}{$\begin{array}{c}\text { Posto } \\
06\end{array}$} & \multicolumn{2}{|c|}{$\begin{array}{c}\text { Posto } \\
07\end{array}$} & \multicolumn{2}{|c|}{$\begin{array}{c}\text { Posto } \\
08\end{array}$} & \multirow{2}{*}{$\begin{array}{c}\text { Mais } \\
\text { preferido }\end{array}$} \\
\hline & \multicolumn{2}{|c|}{ P07 } & \multicolumn{2}{|c|}{ P04 } & \multicolumn{2}{|c|}{ P01 } & \multicolumn{2}{|l|}{ P06 } & P08 & \multicolumn{2}{|r|}{ P05 } & \multicolumn{2}{|c|}{ P02 } & \multicolumn{2}{|c|}{ P03 } & \\
\hline \multicolumn{17}{|c|}{ b) novo ordenamento } \\
\hline \multirow{4}{*}{$\begin{array}{c}\text { Menos } \\
\text { preferido }\end{array}$} & \multicolumn{15}{|c|}{ Posto } & \multirow{4}{*}{$\begin{array}{c}\text { Mais } \\
\text { preferido }\end{array}$} \\
\hline & 01 & 02 & 03 & 04 & 05 & 06 & 07 & 08 & 09 & 10 & 11 & 12 & 13 & 14 & 15 & \\
\hline & $\mathrm{P}$ & & $\mathrm{P}$ & & $\mathrm{P}$ & $\mathrm{P}$ & & & $\mathrm{P}$ & & $\mathrm{P}$ & $\mathrm{P}$ & & & $\mathrm{P}$ & \\
\hline & 07 & & 04 & & 01 & 06 & & & 08 & & 05 & 02 & & & 03 & \\
\hline
\end{tabular}

Figura 2 - Modificação da escala. (a) ordenamento proposto pelo entrevistado, (b) ordenamento modificado.

O Item 3 apresenta a metodologia utilizada para a verificação da existência deste ordenamento utilizando técnicas de simulação e amostragem com probabilidade uniforme.

\section{Metodologia}

Em vista do exposto no Item 1, foram criados cenários com 4, 5, 6 e 7 atributos, possuindo 2, 3 ou 4 níveis cada, formando um total de 12 cenários. Foram criados estímulos com base em planejamentos ortogonais, sendo o número de produtos em cada cenário apresentado na Tabela 2.

\begin{tabular}{|c|c|c|c|}
\cline { 2 - 4 } \multicolumn{1}{c|}{} & \multicolumn{3}{|c|}{ Níveis } \\
\hline Fatores & 2 & 3 & 4 \\
\hline $\mathbf{4}$ & $8(3)$ & $15(6)$ & $16(3)$ \\
\hline $\mathbf{5}$ & $8(2)$ & $15(4)$ & $25(9)$ \\
\hline $\mathbf{6}$ & $8(1)$ & $18(5)$ & $25(6)$ \\
\hline $\mathbf{7}$ & $12(4)$ & $18(3)$ & $32(10)$ \\
\hline
\end{tabular}

Tabela 2 - Número de produtos utilizados em cada cenário considerado. O número entre parênteses corresponde ao número de graus de liberdade.

Na primeira etapa, visando determinar a existência de uma configuração de postos capaz de aumentar o coeficiente de determinação e reduzir a discordância entre os ordenamentos (proposto e predito), foram gerados 1000 ordenamentos aleatórios válidos dos produtos em cada cenário, utilizando uma distribuição uniforme e com reposição Para que estes ordenamentos fossem considerados como respostas válidas impôs-se um coeficiente de determinação mínimo de 0,7 . Cada ordenamento considerado válido foi reordenado dentro da nova escala utilizando amostragem dos ordenamentos possíveis (distribuição uniforme e com reposição), até o limite de 500 tentativas. Cada reordenamento amostrado foi submetido à regressão com variáveis dummy, determinando-se as utilidades parciais e totais e obtendo-se, então, o ordenamento predito. Uma vez encontrado um reordenamento que, simultaneamente, aumentasse o coeficiente de determinação e reduzisse a distância quadrática de postos, interrompia-se o algoritmo de busca e uma nova resposta válida era gerada. A distância quadrática de postos (DQP) foi calculada como o somatório da diferença entre as posições ocupadas por cada produto no ordenamento proposto e no ordenamento predito pelo modelo.

Na segunda etapa, visando determinar se havia uma melhora com o aumento do número de tentativas, fixou-se que para cada resposta válida seriam pesquisados 500 ordenamentos aleatoriamente, registrando-se o melhor ordenamento obtido na nova escala. 
$\mathrm{Na}$ terceira etapa, com o objetivo de verificar qual a proporção de ordenamentos não-válidos $\left(\mathrm{R}^{2}<0,7\right)$ são transformados em ordenamentos válidos $\left(\mathrm{R}^{2}>0,7\right)$ usando a metodologia proposta, foram gerados 1000 ordenamentos aleatórios com $0,4<\mathrm{R}^{2}<0,7$. Cara um destes ordenamentos teve sua escala modificada utilizando uma amostra aleatória de tamanho 500, sendo registrado aquele reordenamento que maximizava $\mathrm{R}^{2}$ e minimizava a DQP.

O Item seguinte apresenta e discute os resultados obtidos.

\section{Resultados e discussão}

A Tabela 1 apresenta os resultados obtidos para a Etapa 1, em que os valores entre parênteses representam o intervalo de $95 \%$ de confiança para a média ou proporção. Como era de se esperar, verificou-se que quanto maior a proporção entre o número de graus de liberdade e o número de parâmetros estimados, menor é a proporção de respostas que são consideradas válidas na população $\left(\mathrm{p}_{\mathrm{R}^{2}>0,7}\right)$, sendo verificada uma relação praticamente linear. No cenário com maior proporção de graus de liberdade (cenário com quatro fatores e três níveis) apenas $25,4 \%$ das respostas foram consideradas válidas. No cenário com 6 fatores e 2 níveis, por sua vez, a proporção de respostas válidas foi de $89,2 \%$, devido ao fato deste cenário apresentar apenas um grau de liberdade.

\begin{tabular}{|c|c|c|c|c|c|c|c|}
\hline \multirow[t]{2}{*}{ Fatores } & \multirow{2}{*}{$\begin{array}{c}\text { Níveis } \\
\text { (produtos) }\end{array}$} & \multirow{2}{*}{$p_{R 2>0,7}$} & \multirow{2}{*}{$\mathbf{p}_{\text {melhora }}$} & \multicolumn{2}{|c|}{ Ordenamento Original } & \multicolumn{2}{|c|}{$\begin{array}{c}\text { Ordenamento utilizando a } \\
\text { nova escala }\end{array}$} \\
\hline & & & & $\mathbf{R}^{2}$ & DQP & $\mathbf{R}^{2}$ & DQP \\
\hline \multirow{3}{*}{4} & $2(8)$ & $\begin{array}{c}0,339 \\
( \pm 0,016)\end{array}$ & $\begin{array}{c}0,202 \\
( \pm 0,014)\end{array}$ & $\begin{array}{c}0,836 \\
( \pm 0,005)\end{array}$ & $\begin{array}{c}8,1 \\
( \pm 0,3)\end{array}$ & $\begin{array}{c}0,858 \\
( \pm 0,005)\end{array}$ & $\begin{array}{c}6,7 \\
( \pm 0,3)\end{array}$ \\
\hline & $3(15)$ & $\begin{array}{c}0,254 \\
( \pm 0,014)\end{array}$ & $\begin{array}{c}0,252 \\
( \pm 0,014)\end{array}$ & $\begin{array}{c}0,790 \\
( \pm 0,004)\end{array}$ & $\begin{array}{c}108,6 \\
( \pm 2)\end{array}$ & $\begin{array}{c}0,812 \\
( \pm 0,004)\end{array}$ & $\begin{array}{l}98,5 \\
( \pm 1,9)\end{array}$ \\
\hline & $4(16)$ & $\begin{array}{c}0,769 \\
( \pm 0,023)\end{array}$ & $\begin{array}{c}0,767 \\
( \pm 0,023)\end{array}$ & $\begin{array}{c}0,861 \\
( \pm 0,005)\end{array}$ & $\begin{array}{l}144,9 \\
( \pm 2,7)\end{array}$ & $\begin{array}{c}0,878 \\
( \pm 0,005) \\
\end{array}$ & $\begin{array}{l}130,7 \\
( \pm 2,6) \\
\end{array}$ \\
\hline \multirow{3}{*}{5} & $2(8)$ & $\begin{array}{c}0,609 \\
( \pm 0,023)\end{array}$ & $\begin{array}{c}0,393 \\
( \pm 0,023)\end{array}$ & $\begin{array}{c}0,866 \\
( \pm 0,006)\end{array}$ & $\begin{array}{c}6,6 \\
( \pm 0,3)\end{array}$ & $\begin{array}{c}0,891 \\
( \pm 0,005)\end{array}$ & $\begin{array}{c}5,0 \\
( \pm 0,3)\end{array}$ \\
\hline & $3(15)$ & $\begin{array}{c}0,575 \\
( \pm 0,023)\end{array}$ & $\begin{array}{c}0,573 \\
( \pm 0,023)\end{array}$ & $\begin{array}{c}0,831 \\
( \pm 0,005)\end{array}$ & $\begin{array}{l}99,0 \\
( \pm 2,0)\end{array}$ & $\begin{array}{c}0,852 \\
( \pm 0,004)\end{array}$ & $\begin{array}{l}88,7 \\
( \pm 1,9)\end{array}$ \\
\hline & $4(25)$ & $\begin{array}{c}0,327 \\
( \pm 0,017) \\
\end{array}$ & $\begin{array}{c}0,327 \\
( \pm 0,017)\end{array}$ & $\begin{array}{c}0,777 \\
( \pm 0,003)\end{array}$ & $\begin{array}{l}654,4 \\
( \pm 8,7)\end{array}$ & $\begin{array}{c}0,791 \\
( \pm 0,003)\end{array}$ & $\begin{array}{r}620,2 \\
( \pm 8,4)\end{array}$ \\
\hline \multirow{3}{*}{6} & $2(8)$ & $\begin{array}{c}0,892 \\
( \pm 0,018)\end{array}$ & $\begin{array}{c}0,539 \\
( \pm 0,029)\end{array}$ & $\begin{array}{c}0,908 \\
( \pm 0,006)\end{array}$ & $\begin{array}{c}4,2 \\
( \pm 0,3)\end{array}$ & $\begin{array}{c}0,934 \\
( \pm 0,005)\end{array}$ & $\begin{array}{c}2,3 \\
( \pm 0,2)\end{array}$ \\
\hline & $3(18)$ & $\begin{array}{c}0,583 \\
( \pm 0,023)\end{array}$ & $\begin{array}{c}0,583 \\
( \pm 0,023)\end{array}$ & $\begin{array}{c}0,814 \\
( \pm 0,004)\end{array}$ & $\begin{array}{l}181,6 \\
( \pm 3,3)\end{array}$ & $\begin{array}{c}0,831 \\
( \pm 0,004)\end{array}$ & $\begin{array}{l}167,1 \\
( \pm 3,1)\end{array}$ \\
\hline & $4(25)$ & $\begin{array}{c}0,689 \\
( \pm 0,024)\end{array}$ & $\begin{array}{c}0,689 \\
( \pm 0,024)\end{array}$ & $\begin{array}{c}0,812 \\
( \pm 0,004)\end{array}$ & $\begin{array}{l}636,5 \\
( \pm 8,5)\end{array}$ & $\begin{array}{c}0,825 \\
( \pm 0,004)\end{array}$ & $\begin{array}{l}602,2 \\
( \pm 8,3)\end{array}$ \\
\hline \multirow{3}{*}{7} & $2(12)$ & $\begin{array}{c}0,422 \\
( \pm 0,020)\end{array}$ & $\begin{array}{c}0,407 \\
( \pm 0,020)\end{array}$ & $\begin{array}{c}0,817 \\
( \pm 0,005)\end{array}$ & $\begin{array}{c}28,3 \\
( \pm 0,9)\end{array}$ & $\begin{array}{c}0,842 \\
( \pm 0,004)\end{array}$ & $\begin{array}{c}23,9 \\
( \pm 0,8)\end{array}$ \\
\hline & $3(18)$ & $\begin{array}{c}0,860 \\
( \pm 0,020)\end{array}$ & $\begin{array}{c}0,859 \\
( \pm 0,020)\end{array}$ & $\begin{array}{c}0,865 \\
( \pm 0,005)\end{array}$ & $\begin{array}{l}157,4 \\
( \pm 3,3)\end{array}$ & $\begin{array}{c}0,880 \\
( \pm 0,005)\end{array}$ & $\begin{array}{l}143,8 \\
( \pm 3,1)\end{array}$ \\
\hline & $4(32)$ & $\begin{array}{c}0,450 \\
( \pm 0,021)\end{array}$ & $\begin{array}{c}0,450 \\
( \pm 0,021)\end{array}$ & $\begin{array}{c}0,777 \\
( \pm 0,003)\end{array}$ & $\begin{array}{l}1456,8 \\
( \pm 13,8)\end{array}$ & $\begin{array}{c}0,789 \\
( \pm 0,003)\end{array}$ & $\begin{array}{l}1403,6 \\
( \pm 13,7)\end{array}$ \\
\hline
\end{tabular}

Tabela 3 - Resultados obtidos aplicando a metodologia descrita com Etapa 1.

Em todos os cenários estudados houve uma redução da DQP e um aumento do $\mathrm{R}^{2}$, estatisticamente comprovados utilizando a análise de variância aplicada aos valores obtidos antes e após a mudança da escala. A proporção de casos melhorados ( $\mathrm{p}_{\text {melhora }}$ ) foi praticamente a mesma proporção de casos considerados válidos na população, exceto para os cenários com 8 produtos. Isto ocorreu porque o número de reordenamentos possíveis na nova escala proposta é de apenas 1716, o que reduz a probabilidade de que exista pelo menos um ordenamento que seja considerado melhor segundo os parâmetros estabelecidos neste 
trabalho. Para efeito de comparação, em um cenário com 12 produtos, o número de reordenamentos possíveis na nova escala é de 352716.

A Tabela 4 apresenta os resultados obtidos nas Etapas 1 e 2. Em todos os cenários estudados houve uma redução na DQP e um aumento no $\mathrm{R}^{2}$ decorrente da busca exaustiva (500 tentativas) aplicada na Etapa 2. Isto comprova que a mudança de escala pode ser tratada como um problema de otimização e que pode-se obter resultados ainda melhores com um aumento no número de buscas. Porém, um tipo de busca como o utilizado neste trabalho é um processo lento. É desejável então que se reescreva o problema aqui proposto como uma função multiobjetivo e aplique-se um método de otimização combinatória para acelerar e tornar o processo de busca mais eficiente.

\begin{tabular}{cccccc}
\hline \multirow{2}{*}{ Fatores } & \multirow{2}{*}{ Níveis } & \multicolumn{2}{c}{ Ordenamento ótimo obtido na Etapa 1 } & \multicolumn{2}{c}{ Ordenamento ótimo obtido na Etapa 1 } \\
\cline { 2 - 5 } & & \multicolumn{1}{c}{$\mathbf{R}^{\mathbf{2}}$} & $\mathbf{D Q P}$ & $\mathbf{R}^{\mathbf{2}}$ & $\mathbf{D Q P}$ \\
\hline \multirow{2}{*}{4} & 2 & $0,858( \pm 0,005)$ & $6,7( \pm 0,3)$ & $0,865( \pm 0,005)$ & $6,4( \pm 0,2)$ \\
& 3 & $0,812( \pm 0,004)$ & $98,5( \pm 1,9)$ & $0,837( \pm 0,004)$ & $86,5( \pm 1,7)$ \\
& 4 & $0,878( \pm 0,005)$ & $130,7( \pm 2,6)$ & $0,899( \pm 0,004)$ & $112,1( \pm 2,4)$ \\
\hline \multirow{2}{*}{5} & 2 & $0,891( \pm 0,005)$ & $5,0( \pm 0,3)$ & $0,898( \pm 0,005)$ & $4,4( \pm 0,2)$ \\
& 3 & $0,852( \pm 0,004)$ & $88,7( \pm 1,9)$ & $0,871( \pm 0,004)$ & $78,8( \pm 1,7)$ \\
& 4 & $0,791( \pm 0,003)$ & $620,2( \pm 8,4)$ & $0,808( \pm 0,003)$ & $575,5( \pm 8,1)$ \\
\hline \multirow{2}{*}{6} & 2 & $0,934( \pm 0,005)$ & $2,3( \pm 0,2)$ & $0,944( \pm 0,005)$ & $1,8( \pm 0,2)$ \\
& 3 & $0,831( \pm 0,004)$ & $167,1( \pm 3,1)$ & $0,850( \pm 0,004)$ & $152,2( \pm 2,8)$ \\
& 4 & $0,825( \pm 0,004)$ & $602,2( \pm 8,3)$ & $0,845( \pm 0,004)$ & $555,6( \pm 7,8)$ \\
\hline \multirow{2}{*}{7} & 2 & $0,842( \pm 0,004)$ & $23,9( \pm 0,8)$ & $0,866( \pm 0,004)$ & $19,5( \pm 0,7)$ \\
& 3 & $0,880( \pm 0,005)$ & $143,8( \pm 3,1)$ & $0,899( \pm 0,004)$ & $128,7( \pm 2,8)$ \\
& 4 & $0,789( \pm 0,003)$ & $1403,6( \pm 13,7)$ & $0,801( \pm 0,003)$ & $1344,2( \pm 13,3)$ \\
\hline
\end{tabular}

Tabela 4 - Resultados obtidos aplicando a metodologia descrita como Etapas 1 e 2.

A Tabela 5 mostra os resultados obtidos de acordo com a Etapa 3. A mudança de escala em todos os cenários estudados proporcionou a modificação de respostas consideradas inválidas $\left(\mathrm{R}^{2}<0,7\right)$ em respostas válidas $\left(\mathrm{R}^{2}>0,7\right)$, sendo que esta modificação variou de $7,0 \%$ à $39,2 \%$. Em relação à população de respostas possíveis, com a modificação de escala pode-se incluir até $12,9 \%$ da população de respostas que são consideradas inválidas.

\begin{tabular}{cccc}
\hline Fatores & $\begin{array}{c}\text { Níveis } \\
\text { (produtos) }\end{array}$ & $\mathrm{P}_{0,4<\mathrm{R} 2<0,7}$ & $\begin{array}{c}\mathrm{P}_{\mathrm{R} 2>0,7} \text { após } \\
\text { mudança da escala }\end{array}$ \\
\hline \multirow{2}{*}{4} & $2(8)$ & 0,389 & 0,184 \\
& $3(15)$ & 0,540 & 0,195 \\
& $4(16)$ & 0,201 & 0,362 \\
\hline \multirow{2}{*}{5} & $2(8)$ & 0,272 & 0,205 \\
& $3(15)$ & 0,379 & 0,339 \\
& $4(25)$ & 0,661 & 0,179 \\
\hline \multirow{2}{*}{6} & $2(8)$ & 0,082 & 0,007 \\
& $3(18)$ & 0,408 & 0,255 \\
7 & $4(25)$ & 0,296 & 0,283 \\
\hline & $2(12)$ & 0,446 & 0,257 \\
& $3(18)$ & 0,157 & 0,392 \\
& $4(32)$ & 0,518 & 0,171 \\
\hline
\end{tabular}

Tabela 5- Resultados obtidos aplicando a metodologia descrita na Etapa 3. 
Os resultados mostram que a modificação de escala proposta neste trabalho pode ser uma ferramenta adequada para o tratamento posterior dos dados obtidos em um estudo utilizando análise conjunta, especialmente quando o número de entrevistados é pequeno ou a coerência do modelo obtido é baixa. Porém, estudos devem ser realizados para determinar o efeito da modificação da escala sobre as utilidades e importâncias calculados. Este estudo pode ser realizado por simulação, construindo-se uma população com importâncias e utilidades conhecidas e aplicando a regressão para estimação destes valores antes e após a modificação da escala. Tais estudos já estão em andamento.

\section{Conclusões}

Os resultados obtidos mostram que a modificação da escala de postos promove um aumento do coeficiente de correlação da regressão com variáveis dummy e uma redução da distância quadrática média dos postos, que são duas medidas da qualidade do modelo de utilidades, para todos os cenários utilizados neste trabalho. A extensão desta melhora foi maior quando maior foi o número de buscas de um novo ordenamento. Isso indica que trata-se de um problema de otimização combinatória, e métodos específicos devem ser utilizados para acelerar o processo de obtenção da ordenação ótima.

Utilizando esta técnica pode-se incluir respondentes cujo ordenamento tenha sido considerado inválido sob um dado critério ( $\mathrm{R}^{2}$ mínimo, por exemplo) e, portanto, aumentar o tamanho da amostra sobre a qual serão calculadas as importâncias e utilidades da população. Porém, para a aplicação desta modificação, devem ser feitos estudos comparativos sobre uma população conhecida, para verificar o efeito da mudança da escala na estimação das utilidades e importâncias.

\section{Referências Bibliográficas}

AUTY, S. (1995) Using conjoint analysis in industrial marketing: The role of judgment. Industrial Marketing Management. 24(3):191-206.

DARMON, R. Y. e ROUZIÈS, D. (1999) Internal Validity of Conjoint Analysis Under Alternative Measurement Procedures. Journal of Business Research. 46(1):67-81.

GREEN, P. e SRINIVASAN, V. (1990) Conjoint Analysis in Marketing: New Developments with implications for Research and Practice. Journal of Marketing. 54:3-19.

LAMBIN, J. J. Marketing Estratégico. 4 ed. Amadora: McGraw-Hill, 2000, 756 p.

MALHOTRA, N. K. Pesquisa de Marketing - Uma Orientação Aplicada. 3 ed. Porto Alegre: Bookman, 2001, $720 \mathrm{p}$.

MOORE, W. L., LOUVIERE, J. J. e VERMA, R. (1999) Using conjoint analysis to help design product platforms. Journal of Product Innovation Management. 16(1):27-39.

NÆS, T., KUBBERØD, E. e SIVERTSEN, H. (2001) Identifying and interpreting market segments using conjoint analysis. Food Quality and Preference. 12(2):133-143.

PARK, C. S. (2003) The robustness of hierarchical Bayes conjoint analysis under alternative measurement scales. Journal of Business Research. In Press, Corrected Proof.

REUTTERER, T. e KOTZAB, H. W. (2000) The Use of Conjoint-Analysis for Measuring Preferences in Supply Chain Design. Industrial Marketing Management. 29(1):27-35.

WITTINK, D. R., KRISHNAMURTHI, L. e NUTTER, J. (1982) Comparing Derived Importance Weights Across Attributes. Journal of Consumer Research. 8:471-474.

WITTINK, D. R., VRIENS, M. e BURHENNE, W. (1994) Commercial use of conjoint analysis in Europe: Results and critical reflections. International Journal of Research in Marketing. 11(1):41-52. 